Chapter 11

\title{
Regulation of Autophagy by \\ Short Chain Fatty Acids in Colon Cancer Cells
}

\author{
Djamilatou Adom and Daotai Nie \\ Additional information is available at the end of the chapter \\ http://dx.doi.org/10.5772/54999
}

\section{Introduction}

Short chains fatty (SCFAs) acids are organic fatty acids that are the major products of bacterial fermentation of undigested dietary fiber and resistant starch in the colon. Propionate, acetate and butyrate are the main SCFAs produced from fermentation and serve as fuel for colonocytes. SCFAs serve as regulators of intracellular $\mathrm{pH}$, cell volume, and other functions associated with ion transport. Moreover, SCFAs act as regulators of proliferation, differentiation and gene expression. Our recent studies reported that SCFAs promote autophagy in colon cancer cells. In this chapter, the regulation of autophagy by short chain fatty acids in colon cancer cells will be discussed in details including the mechanism of action.

\section{Short chain fatty acids and the colon}

Short chain fatty acids are organic fatty acids with 1 to 6 carbon atoms and the major products of bacterial fermentation in the human large intestine. Those are mostly derived from polysaccharides, oligosaccharides, proteins, peptides and glycoproteins precursors by anaerobic microorganisms [1]. Diets high in fiber, resistant starches and complex carbohydrates lead to an increase in the levels of SCFAs. The principal SCFA involved in mammalian physiology are acetate $\mathrm{c} 2$, propionate $\mathrm{c} 3$ and butyrate $\mathrm{c} 4$. Formate, valerate, caproate, lactate and succinate are other fermentation products which are produced but to a lesser extent [2]. The scope of this chapter will be limited to propionate, butyrate and acetate.

In the colon, short chain fatty acids are absorbed at the same time as sodium and water absorption. Two mechanisms of absorption have been proposed. During diffusion of protonated SCFAs, luminal protons $\left(\mathrm{Na}^{+} / \mathrm{H}^{+}\right.$exchange, $\mathrm{K}^{+} \mathrm{H}^{+}$-ATPase or bacterial metabolic activity) 
acidify the colonic lumen. This creates a $\mathrm{pH}$ between the colonic lumen compared to the systemic circulation. This $\mathrm{pH}$ gradient can promote the diffusive movement of SCFAs. With anion exchange, a family of anion exchangers mediates SCFAs and HCO3 exchange and entry across the membrane[3]. Once absorbed, SCFAs are used preferentially as fuel for colonic epithelial cells [4]. Butyrate is used preferentially over propionate and acetate. Those SCFAs are later transported to the liver. There, propionate acts as a substrate for gluconeogenesis and inhibits cholesterol synthesis in hepatic tissue. Acetate is utilized in the synthesis of long chain fatty acids, glutamine, glutamate and beta-hydroxybutyrate [2, 4]. Over the last a few decades, understanding the role of short chain fatty acids in the gastrointestinal physiology has grown considerably. They are attractive because of their role as potential therapeutic agents in diversion colitis, ulcerative colitis, radiation proctitis, pouchitis and antibiotic- associated diarrhea [4]. Furthermore, SCFAs have anti-tumor activities in colon cancer by promoting autophagy and apoptosis. In the next section, the role of SCFAs in cancer, mostly in the colon will be discussed.

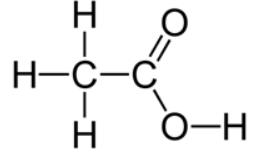

Acetic acid (acetate)

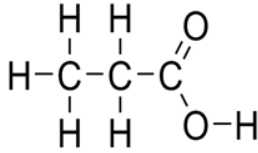

Propionic acid (propionate)

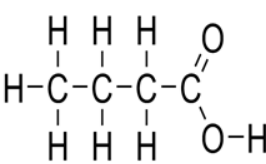

Butyric acid (butyrate)

Figure 1. Nomenclature of short chain fatty acids (SCFAs). Different SCFAs (acetate, propionate and butyrate) are illustrated in this figure.

\section{The role of short chain fatty acids in colon cancer}

Diet has a considerable influence on the risk of colon cancer. A diet high in fat has been considered to promote colon cancer while increased fiber and complex carbohydrates in the diet may protect against colon cancer. Butyrate is believed to be mostly responsible for the tumor inhibitory effects of dietary fiber. Sodium butyrate is known to be an effective inducer of cell differentiation. Colorectal cancer cells treated with sodium butyrate showed a more differentiated cell state $[5,6]$. It is believed that the protective effect of dietary fibers is associated with butyrate production in the colon which possibly decreases the occurrence of neoplasia in colonocytes. For example, in reference [7], fibers associated with high butyrate were protective against colon cancer. Patients with familial polyposis (FAP) syndrome develop hundreds to thousands of begin tumors of the colon, some of which will progress to colon cancer if not removed. These FAP patients produced less butyrate than healthy controls and patients without polyps. All the studies suggest that butyrate has a protective effect against colon cancer. In the next section, different mechanisms of action of short chain fatty acids on colon cancer will be discussed. 


\section{Mechanisms of action of short chain fatty acids on colon cancer cells}

Different mechanisms of action has been proposed through which sodium butyrate regulate different genes to exert an inhibitory effect on colon cancer development. Sodium butyrate induces growth inhibition in colon cancer cells by promoting histone hyperacetylation and induction of the cell cycle inhibitor p21 [8]. Other short chain fatty acids such as propionate and valerate were also shown to inhibit cell tumor cells but to a lesser extent than butyrate. However for acetate, no effects were observed on cell proliferation when it was used at a concentration of $20 \mathrm{mM}$ [9]. Cells other than colonocytes, mainly smooth muscle cells are also present in the colon. The effect of SCFAs on colon smooth muscle cells was quiet different. Sodium butyrate promotes the proliferation of smooth muscle cells. Propionate also promotes colon smooth muscle cells proliferation to a lesser extent than butyrate while acetate had no effects [9]. The cell growth inhibitory effect of butyrate on colon cancer cells is attributed to its ability to induce histone hyperacetylation through inhibition of histone deacetylase (HDAC). Histone hyperacetylation usually results in relaxation of chromatin, thus making DNA more accessible to transcription factors. For example in reference [10], the cell cycle inhibitor p21 gene was increased and involved in the butyrate effect on colon cancer cell proliferation. The promoter region of p21 was shown to harbor butyrate-responsive elements. Upon butyrate treatment, p21 was induced due to HDAC inhibition resulting in G1 phase arrest [9, 11-13].

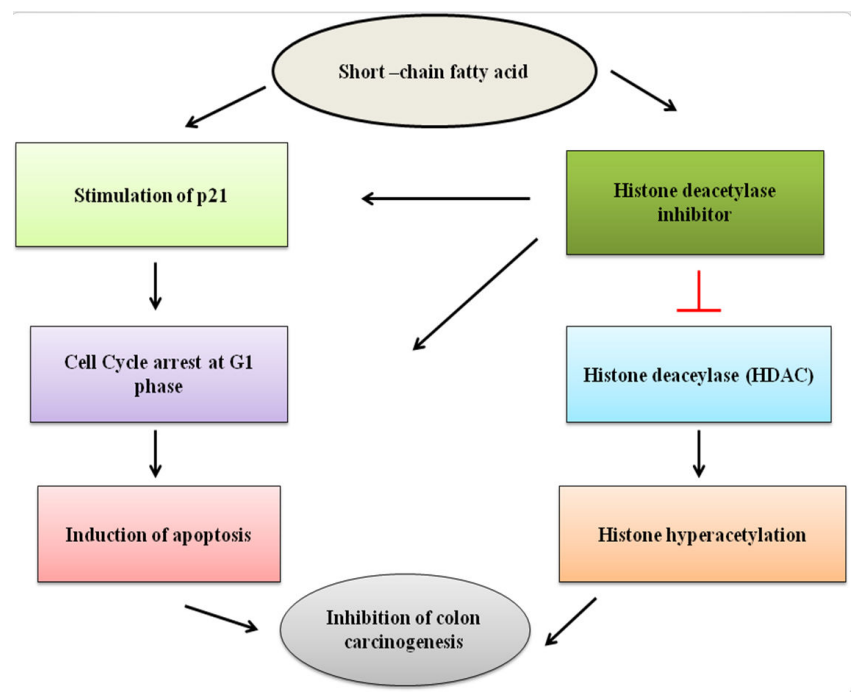

Figure 2. Mechanism of inhibition of colon cancer cells by short chain fatty acids. Short chain fatty acids, particularly butyrate can inhibit colon cancer by different mechanisms. In this figure, treatment of colon cancer cells with butyrate leads to the activation of the cycle inhibitor p21, followed by cell cycle arrest at G1 phase and induction of apoptosis. Moreover, SCFAs treatment can promote the inhibition of histone deacetylase, leading to histone hyperacetylation and availability of chromatin structure for binding by different transcription factors. Both histone hyperacetylation and cell cycle protein p21 inhibit colon cancer growth. 
Sodium butyrate also inhibits colon cancer cell invasion by activating tissue inhibitor matrix metalloproteinase (TIM) 1- and 2, thus inhibiting the activity of metalloproteinases (MMPs). Furthermore, sodium butyrate reduces the adherence of colon cancer cells to the basement membrane protein laminin substrate via fibronection or type IV collagen resulting in the inhibition of cancer growth. Inflammatory cytokines such as IL- 4 and TNF- $\alpha$ also play a role in the inhibitory role of butyrate in colon cancer [14]. G-protein coupled receptors are also found to play a role on colon cancer and their role will be discussed in the next section.

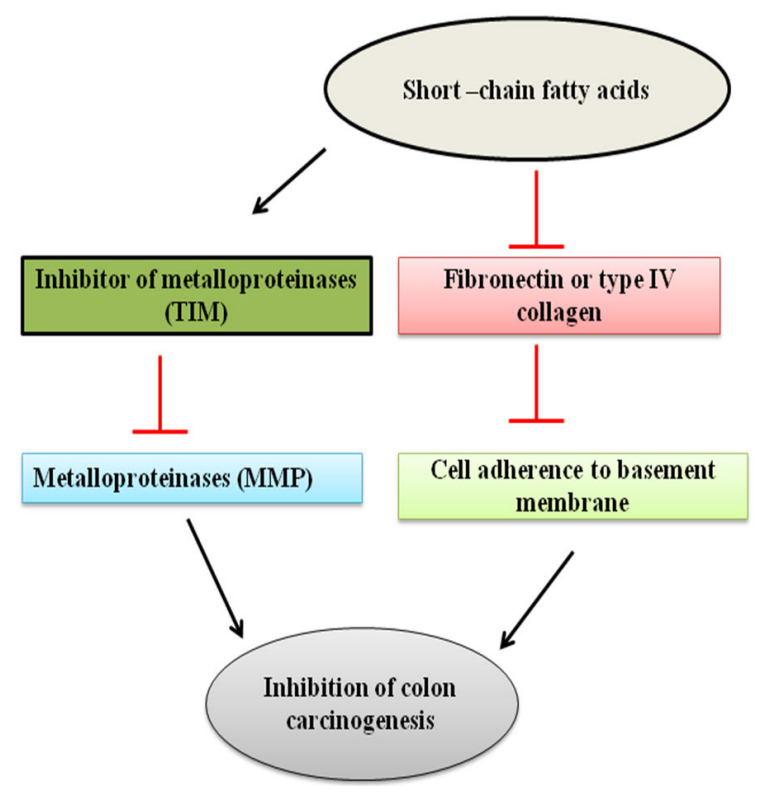

Figure 3. Other mechanisms used by short chain fatty acids to inhibit colon cancer carcinogenesis. Treatment with SCFAs can inhibit the destruction of the basement membrane through the stimulation of the inhibitor of metalloproteinases (TIM). TIM will inhibit metalloproteinases and prevent them from destroying the basement membrane. SCFAs also inhibit the adherence of colon cancer cells to the basement membrane by reducing fibronection of type IV collagen levels.

\section{The roles of short chain fatty acid receptors GPR41 and GPR43 on colonic functions}

G-protein coupled receptors (GPCRs) consist of a large and diverse family of proteins that mainly transduce extracellular stimuli to intracellular signals. GPRC family is among the largest and more diverse family of proteins in the mammalian genome and contain 7 spanning membrane helixes, an extracellular N-terminus and an intracellular C-terminus. GPCRs can be coupled by at least $18 \mathrm{G} \alpha$ which forms a heterodimer with $\mathrm{G} \beta$ subunits, which have at least 5 types, and $G \gamma$ subunit, of which there are at least 11 types. Over 800 GPCRs have been 
identified in the human genome. The family of GPCR protein is activated upon binding of a ligand or agonist on the extracellular $\mathrm{N}$-terminus that leads to a conformational change and activation of the G-protein heterodimer. At least 50 GPCRs have unknown ligands and are referred as orphans. Depending on the type of GPCRs that is being activated, diverse downstream signaling will be activated.GPRCs respond to different stimuli such as light, neurotransmitters, amino acids, hormones and activate different signaling pathways $[15,16]$.

Recently, short chain fatty acids (acetate, propionate and butyrate) were reported as ligands for two orphan GPRCs, GPR41 and GPR43. GPR43 is expressed in immune cells whereas GPR41 is present in blood vessel endothelial cells, particularly in adipose tissue with significant expression also in immune cells and endothelial cells of other tissues [17]. Both GPR41 and GPR43 are expressed in colonic mucosa suggesting their role in the normal development or functions of the colon tissue [16]. A study by Tang and al. revealed more information on the function of GPR43 in colon cancer. Immunohistochemistry showed a reduction of GPR43 in human colon cancers compared to normal human colon tissues. No epigenetic changes such as promoter hypermethylation or chromatin compaction due to histone deacetylation (HDAC) were found responsible for the repression or silencing of GPR43 in colon cancer. In order to determine the function of GPR43 in colon cancer, GPR43 was restored in colon cancer cells. Treatment of those GPR43 expressing cells with short chain fatty acids propionate and butyrate rendered the cells more sensitive, caused cell death and promoted cell cycle arrest at Go/G1 phase. The study suggested that loss of GPR43 expression may contribute to colon cancer development and progression [18]. In the next section, the role of short chain fatty acids in the fate of colon cancer cells will be discussed.

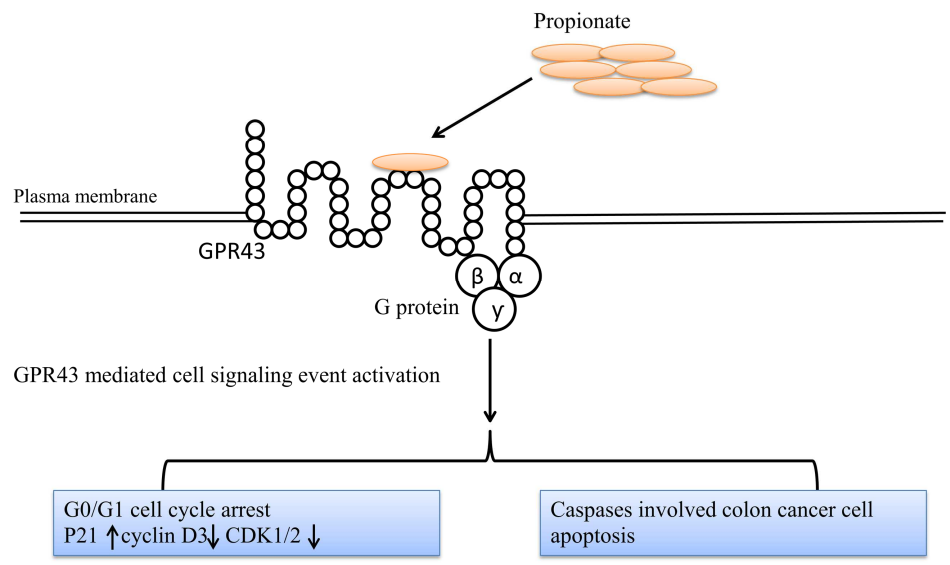

Figure 4. Mechanism of GPR43 role as a tumor suppressor in colon cancer. Treatment of colon cancer cells with SCFAs (propionate) results in binding of the GPR43 on the extracellular surface. This leads to the activation of intracellular downstream signaling such as increase in cell cycle protein p21 and CDK1/2 while cyclin D3 is increased resulting in cell cycle arrest at Go/G1 phase. Propionate treatment also stimulates different caspases which activates apoptosis and death of colon cancer cells. 


\section{Regulation of autophagy by short chain fatty acids}

Short chain fatty acids were initially reported to induce apoptosis (type I programmed cell death) in colon cancer. Treatment of colon cancer cells with butyrate inhibited cell proliferation, promoted apoptosis in 79\% of cells through the activation of caspase- 3 and the degradation of PARP [19] [20]. Recently, SCFAs, particularly propionate was reported to induce autophagy as evidenced by an increased LC3 punctuates formation and upregulation of LAMP-2 [21]. In this section, the regulation of autophagy by short chain fatty acids will be discussed. The mechanisms involved will be discussed in details, including the signaling pathways involved.

\subsection{Mechanism of autophagy regulation by short chain fatty acids in colon cancer}

In autophagy studies, it is very well established that the mammalian target of rapamycin (mTOR) negatively regulates autophagy. The autophosphorylation at Ser2481 is regarded as an indicator of its catalytic activity. Post propionate treatment of colon cancer cells HCT116, a strong time dependent reduction in the phosphorylation state at Ser2481 was observed, while there were no changes in the total mTORC levels. Another key downstream effector of mTOR, p70S6K, whose phosphorylation status at Thr389 reflects mTOR activity, whereas phosphorylation at Thr421/Ser424 is thought to activate p70S6K was also performed. Following propionate treatment, a reduced phosphorylation of p70S6K at Thr389 was observed by $7 \mathrm{~h}$ confirming that downregulation of the mTOR signaling pathway is a mechanism for propionate to induce autophagy. The group then hypothesized that propionate must induce mTOR signaling from the inhibition of PI3/Akt pathway, which was shown to activate mTOR in response to the introduction of nutrient and growth factors. However, no changes in the phosphorylation state of Akt at S473 or Thr308 or the total Akt were observed post propionate treatment. Another pathway which acts upstream of the mTOR pathway, the AMP-activated protein kinase (AMPK) [22], an inhibitor of the mTOR protein and a sensor of cellular bioenergetics, was significantly activated.

Interestingly, propionate mediated AMPK activation caused a decrease in ATP levels in colon cancer cells due to a breach of the mitochondrial membrane potential. In more details, propionate depolarized the mitochondrial membrane, which was shown using mitotracker deep red, a dye that stains the mitochondria in live cells and accumulates in proportion to the membrane potential. The proportion of mitochondria with lower fluorescence intensity, which represents the depolarized mitochondria, was increased post propionate treatment in a dose- and time dependent manner in the colon cancer cells treated. The study in references [21, 23] demonstrated that propionate causes mitochondrial defect leading to ATP depletion and release of reactive oxygen species (ROS). Excessive ROS levels have been attributed to induction of autophagy. The defective mitochondria post treatment is removed by a selective autophagy process known as mitophagy. Mitophagy will be discussed in the next section and how it is regulated in colon cancer cells post propionate treatment. 


\subsection{Propionate treatment causes mitophagy in colon cancer cells}

Mitochondria are cells organelles that primarily produce ATP via oxidative phosphorylation in the inner membrane of the mitochondria. During changes in the environment, ATP synthesis can be disrupted leading to the production of reactive oxygen species (ROS) and release of proteins to promote cell death. Several pathologies have impaired mitochondria, oxidative stress, accumulation of protein aggregates and autophagic stress. Oxidative stress can lead to the nonspecific modification of proteins and contributes to protein aggregation. Interestingly, the cell can adopt its own defense mechanism against aberrant mitochondria, which can be harmful to the cell [24]. This mechanism termed mitophagy was first observed in mammalian cells by early electron microscopy studies, where increased mitochondrial sequestration was identified in lysosomes following stimulation of hepatocytes catabolism with glucagon [25]. This selective autophagy process is characterized by the removal of excess or damaged mitochondria in order to prevent activation of apoptotic cell death [26]. Mitophagy has been shown to play a role during cellular quality control. For instance, in yeast and in mammalian cells, mitophagy is preceded by mitochondrial fission, which divides elongated mitochondria into pieces of manageable size for encapsulation and also quality control of segregation of damaged mitochondrial material for selective removal by mitophagy. Another process, mitochondrial fusion, occurs every 5 to 20 minutes and was shown to reduce mitochondrial depolarization in two cell lines (COS7 and INS1). Mitochondrial fission, fusion or mitophagy are all important for mitochondrial homeostasis [27]. Mitophagy has also been shown to be required for steadystate turnover of mitochondria, for the adjustment of mitochondrion numbers to changing metabolic requirements and during specialized developmental stages in mammalians cells such as during red blood cell differentiation [25]. Some important proteins such as ULK1 and ULK2 (two Atg1 homologues), Parkinson's disease genes $\alpha$-synuclein, parkin, PINK1 and DJ-1 are all involved in mitophagy. During the selection of mitochondria for mitophagy, mitochondrial components of the cell are identified by partner cytosolic proteins such as Parkin or Nix that bind to the surface and tag it for degradation [24-26].

Treatment of colon cancers cells HCT116 with propionate resulted in reduced staining intensity of mitochondria and an increased colocalization between mitochondria and punctuates GFP-LC3. COXIV, a mitochondrial marker was also reduced and was localized as defective mitochondria by autolysosomes. An ubiquitin-binding protein-p62, a protein that interacts with LC3 and regulates autophagosome formation, significantly colocalized with mitochondrial COXIV. Flow cytometry analysis showed that most of colon cancer cells treated with propionate showed a reduced Mito Tracker Deep Red staining and enhanced GFP-LC3 fluorescence. Addition of chloroquine, an inhibitor of autophagic degradation, dramatically increased the accumulation of defective mitochondria. All the experiments performed in HCT116 colon cancer cells post treatment suggest that propionate triggers mitophagy. This mitophagy selectively targets mitochondria with a depolorized membrane potential [21, 23]. 


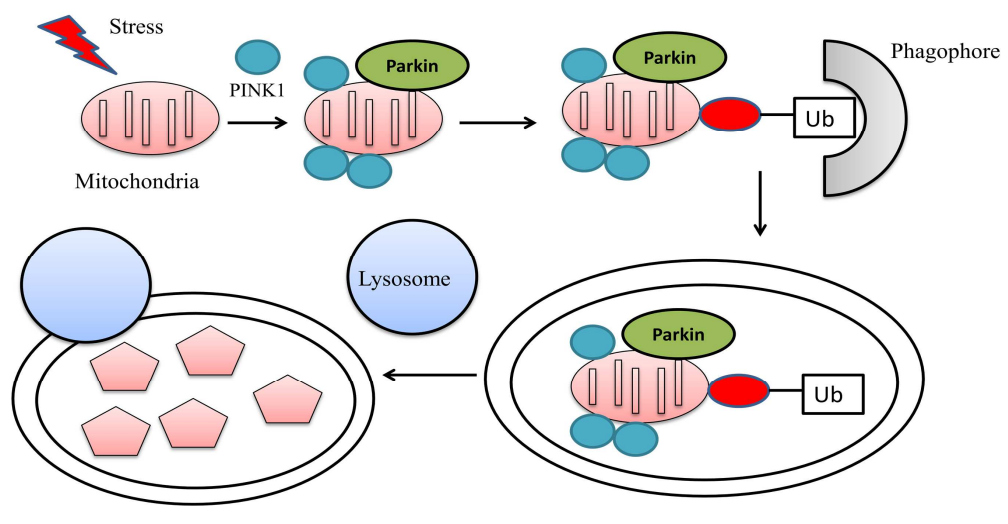

Figure 5. Mechanisms of mitophagy and representation of proposed role of short chain fatty acids on mitochondria. Treatment of colon cancer cells with SCFAs depolarizes and damages the mitochondria. Reduced mitochondrial membrane potential leads to the accumulation of phosphatase and tensin homolog-induced putative kinase 1 (PINK1) and recruitment of the E3 ubiquitin ligase Parkin to mitochondria. Parkin, then promotes the ubiquitination of proteins in the mitochondrial membrane, which targets the damaged mitochondrion for removal by an autophagasome.

\subsection{Short chain fatty acids and lipid metabolism in colon cancer cells}

Interestingly, treatment of HCT116 cells with propionate altered the lipid metabolism. Lipids play an important role in cell structure and metabolism. Post treatment with propionate, the expression of fatty acid synthase, the enzyme that catalyzes the synthesis of long chain-fatty acids (LCFAs) from acetyl-CoA (ACC) and malonyl-CoA, was reduced. Furthermore, GSK-3 $\beta$, which inhibits endergonic glycogen synthesis by phosphorylation and activation of glycogen synthase, was also downregulated. AMPK kinase, which was increased post propionate treatment further phosphorylates and inhibit acetyl-CoA, thus de novo lipid synthesis. The inactivation of ACC by AMPK mediates the increase in mitochondrial import and oxidation of LCFAs, resulting in the generation of ATP.

Energy deprivation can stimulate mitochondrial biogenesis in skeletal muscle in an AMPKdependent manner. When the effects of propionate treatment in mitochondrial biogenesis were investigated, the mRNA levels of nuclear gene mitochondrial transcription factor A (Tfam) and mitochondrial transcription factor B (mtTFB) were stimulated, peaking at 8.5 and $11.5 \mathrm{~h}$ respectively, before returning to the initial pre-stimulatory levels. Other transcription factors related to heme biosynthesis and mitochondria biogenesis named nuclear respiratory factors- 1 and 2 (NRF-1 and NRF-2) and polymerase gamma (pol- $\gamma$ ) expression were also stimulated at the mRNA level in colon cancer cells after propionate treatment. On the other hand, peroxisome proliferator activated receptor- $\gamma$ was reduced. 
Moreover, an increase in mitochondria complex subunits and increase in Mitotracker greem FM fluorescence also indicated and confirmed that HCT116 cells adapt to propionateinduced ATP depletion by downregulating anabolic processes such as glycogen and lipid synthesis, while stimulating mitochondrial biogenesis in an attempt to resume cellular energy homeostatis [21, 23].

\subsection{Propionate, autophagy and apoptosis}

Autophagy and apoptosis are two programmed-cell deaths that may be interconnected and even simultaneously regulated by the same trigger in tumor cells. During apoptosis, cells are destroyed as an end result of caspase mediated destruction of the cellular structure. There exist two core pathways inducing apoptosis: the extrinsic and intrinsic pathways. The extrinsinc pathway is triggered by the Fas death receptor (DR), which depends on the combination of FasL and Fas. The other process, the intrinsic pathway leads to apoptosis upon sensing of an extracellular stimuli or intracellular signal that renders the mitochondrial membrane permeable and releases cytochrome c [28-31].

Molecular pathways leading to cancer can cross-talk. Autophagy and apoptosis can particularly act as partners to induce cell death in a coordinated or cooperative fashion. Although both can be triggered by common upstream signals, this will have different effects on the cell fate. For instance, autophagy can function as a double edged sword to either promote or inhibit cell death. In most cases, inhibition of autophagy leads to an increase susceptibility to apoptotic stimuli [29]. For instance when colon cancer cells were treated with propionate, [21] found that the induction of autophagy has a protective effect on HCT116 cells. SCFAs were previously shown to induce caspase-3-mediated apoptosis. Cotreatment of colon cancer cells (HCT116 and SW480) with 3-methyladenine (3-MA), an inhibitor of autophagy, significantly reduced the percentage of GFP-LC3 formation. However, $12 \mathrm{~h}$ after the initiation of treatment of propionate/3MA, the number of apoptotic cells increased as indicated by the high annexin-V staining. Western blot analysis also revealed increased cleavages of the pro-apoptotic cascapase-7 and executioner caspase-3, which are all critical mediators of the mitochondrial events of apoptosis in cells treated with propionate/3MA compared to nontreated group. Addition of another inhibitor of autophagy, chloroquine, enhanced apoptosis in HCT116 cells, especially at the later stages of treatment. Since depletion of AMPK $\alpha$ using shRNA was also shown to mimic the effects of autophagy silencing,colon cancer cells depleted of the AMPK $\alpha$ were also treated with propionate. AMPK $\alpha$ depleted cells showed a more significant cytotoxicity post propionate treatment. Further depletion of autophagy by knocking down ATG5 expression, an important protein required for autophagy, reduced the ability of propionate to induce GFPLC3 punctae formation, indicating successful depletion. This inhibition of autophagy also confirmed the protective role of autophagy in colon cancer cells post treatment with the short chain fatty acid, propionate. All the findings by [21, 23] suggested that autophagy confers a protective role in propionate-mediated cell death in colon cancer cell. 


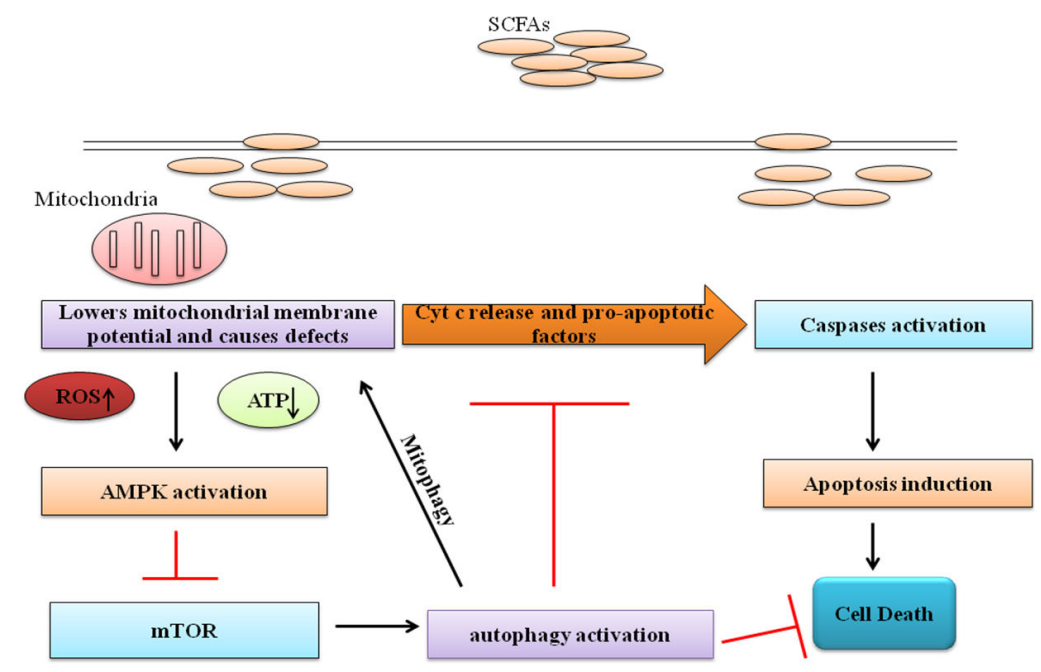

Figure 6. Proposed model for regulation of autophagy by short chain fatty acids in colon cancer cells. Treatment of colon cancer cells with short chain fatty acids increases ROS levels, lowers the mitochondrial membrane potential and ATP levels. This results in a reduction of cellular energy and defects in mitochondria. As a result, downstream signaling pathway AMPK is activated while mTOR is inhibited and resulting in the activation of autophagy. At the same time, defective mitochondria is removed by a process known as mitophagy in order to rescue the cells from apoptosis and cell death. The activation of autophagy has a protective role on the cell as it prevents cell death.

\section{Conclusion}

Short chain fatty acids are the major by-products of bacterial fermentation of undigested fibers in the colon. Short chain fatty acids, particularly propionate has been shown to promote differentiation, growth arrest and apoptosis in colon cancer cells. Furthermore, SCFAs can promote autophagy to retard mitochondrial defect stimulated apoptosis. Although the in vitro studies demonstrated a role of short chain fatty acids in promoting autophagy and protecting the cells from apoptotic death, the relevance of this finding has yet to be investigated in vivo. Moreover, cotreatment of colon cancer cells with SCFAs and chloloroquine, an inhibitor of autophagy may be an effective therapy approach to be investigated.

\section{Author details}

Djamilatou Adom and Daotai Nie*

Department of Medical Microbiology, Immunology, and Cell Biology, Southern Illinois University School of Medicine and Simmons Cancer Institute, Springfield, IL, USA 


\section{References}

[1] Macfarlane S, Macfarlane GT. Regulation of short-chain fatty acid production; 2003.

[2] Cummings JH, Macfarlane GT. The control and consequences of bacterial fermentation in the human colon. The Journal of applied bacteriology. 1991 Jun;70(6):443-59.

[3] Augenlicht LH, Mariadason JM, Wilson A, Arango D, Yang W, Heerdt BG, et al. Short chain fatty acids and colon cancer. The Journal of nutrition. 2002 Dec;132(12): 3804S-8S.

[4] Cook SI, Sellin JH. Review article: short chain fatty acids in health and disease. Alimentary pharmacology \& therapeutics. 1998 Jun;12(6):499-507.

[5] Fu H, Shi YQ, Mo SJ. Effect of short-chain fatty acids on the proliferation and differentiation of the human colonic adenocarcinoma cell line Caco-2. Chinese journal of digestive diseases. 2004;5(3):115-7.

[6] D'Argenio G, Mazzacca G. Short-chain fatty acid in the human colon. Relation to inflammatory bowel diseases and colon cancer. Adv Exp Med Biol. 1999;472:149-58.

[7] McIntyre A, Young GP, Taranto T, Gibson PR, Ward PB. Different fibers have different regional effects on luminal contents of rat colon. Gastroenterology. 1991 Nov; 101(5):1274-81.

[8] Hinnebusch BF, Meng S, Wu JT, Archer SY, Hodin RA. The effects of short-chain fatty acids on human colon cancer cell phenotype are associated with histone hyperacetylation. The Journal of nutrition. 2002 May;132(5):1012-7.

[9] Blottiere HM, Buecher B, Galmiche JP, Cherbut C. Molecular analysis of the effect of short-chain fatty acids on intestinal cell proliferation. The Proceedings of the Nutrition Society. 2003 Feb;62(1):101-6.

[10] Nakano K, Mizuno T, Sowa Y, Orita T, Yoshino T, Okuyama Y, et al. Butyrate activates the WAF1/Cip1 gene promoter through Sp1 sites in a p53-negative human colon cancer cell line. J Biol Chem. 1997 Aug 29;272(35):22199-206.

[11] Archer S, Meng S, Wu J, Johnson J, Tang R, Hodin R. Butyrate inhibits colon carcinoma cell growth through two distinct pathways. Surgery. 1998 Aug;124(2):248-53.

[12] Archer SY, Meng S, Shei A, Hodin RA. p21(WAF1) is required for butyrate-mediated growth inhibition of human colon cancer cells. Proceedings of the National Academy of Sciences of the United States of America. 1998 Jun 9;95(12):6791-6.

[13] Siavoshian S, Segain JP, Kornprobst M, Bonnet C, Cherbut C, Galmiche JP, et al. Butyrate and trichostatin A effects on the proliferation/differentiation of human intestinal epithelial cells: induction of cyclin D3 and p21 expression. Gut. 2000 Apr;46(4): 507-14. 
[14] Andoh A, Tsujikawa T, Fujiyama Y. Role of dietary fiber and short-chain fatty acids in the colon. Current pharmaceutical design. 2003;9(4):347-58.

[15] Kroeze WK, Sheffler DJ, Roth BL. G-protein-coupled receptors at a glance. J Cell Sci. 2003 Dec 15;116(Pt 24):4867-9.

[16] Tazoe H, Otomo Y, Kaji I, Tanaka R, Karaki SI, Kuwahara A. Roles of short-chain fatty acids receptors, GPR41 and GPR43 on colonic functions. J Physiol Pharmacol. 2008 Aug;59 Suppl 2:251-62.

[17] Brown AJ, Goldsworthy SM, Barnes AA, Eilert MM, Tcheang L, Daniels D, et al. The Orphan G protein-coupled receptors GPR41 and GPR43 are activated by propionate and other short chain carboxylic acids. J Biol Chem. 2003 Mar 28;278(13):11312-9.

[18] Tang Y, Chen Y, Jiang H, Robbins GT, Nie D. G-protein-coupled receptor for shortchain fatty acids suppresses colon cancer. Int J Cancer. Feb 15;128(4):847-56.

[19] Ruemmele FM, Schwartz S, Seidman EG, Dionne S, Levy E, Lentze MJ. Butyrate induced Caco-2 cell apoptosis is mediated via the mitochondrial pathway. Gut. 2003 Jan;52(1):94-100.

[20] Ruemmele FM, Dionne S, Qureshi I, Sarma DS, Levy E, Seidman EG. Butyrate mediates Caco-2 cell apoptosis via up-regulation of pro-apoptotic BAK and inducing caspase-3 mediated cleavage of poly-(ADP-ribose) polymerase (PARP). Cell Death Differ. 1999 Aug;6(8):729-35.

[21] Tang Y, Chen Y, Jiang H, Nie D. Short-chain fatty acids induced autophagy serves as an adaptive strategy for retarding mitochondria-mediated apoptotic cell death. Cell Death Differ. Apr;18(4):602-18.

[22] Hardie DG. AMP-activated protein kinase: an energy sensor that regulates all aspects of cell function. Genes Dev. Sep 15;25(18):1895-908.

[23] Tang $Y$, Chen $Y$, Jiang H, Nie D. The role of short-chain fatty acids in orchestrating two types of programmed cell death in colon cancer. Autophagy. Feb;7(2):235-7.

[24] Kubli DA, Gustafsson AB. Mitochondria and mitophagy: the yin and yang of cell death control. Circ Res. Oct 12;111(9):1208-21.

[25] Youle RJ, Narendra DP. Mechanisms of mitophagy. Nat Rev Mol Cell Biol. Jan;12(1): 9-14.

[26] Kim I, Rodriguez-Enriquez S, Lemasters JJ. Selective degradation of mitochondria by mitophagy. Arch Biochem Biophys. 2007 Jun 15;462(2):245-53.

[27] Lee J, Giordano S, Zhang J. Autophagy, mitochondria and oxidative stress: cross-talk and redox signalling. Biochem J. Jan 15;441(2):523-40.

[28] Maiuri MC, Zalckvar E, Kimchi A, Kroemer G. Self-eating and self-killing: crosstalk between autophagy and apoptosis. Nature reviews. 2007 Sep;8(9):741-52. 
[29] Rikiishi H. Novel Insights into the Interplay between Apoptosis and Autophagy. International journal of cell biology.2012:317645.

[30] Eisenberg-Lerner A, Bialik S, Simon HU, Kimchi A. Life and death partners: apoptosis, autophagy and the cross-talk between them. Cell death and differentiation. 2009 Jul;16(7):966-75.

[31] Galluzzi L, Vicencio JM, Kepp O, Tasdemir E, Maiuri MC, Kroemer G. To die or not to die: that is the autophagic question. Current molecular medicine. 2008 Mar;8(2): 78-91. 
